\title{
Effect of Annealing on Mechanical Quality Factor of Fused Quartz Hemispherical Resonator
}

\author{
Mohammed J. Ahamed, Doruk Senkal, and Andrei M. Shkel \\ MicroSystems Laboratory, University of California, Irvine, CA, USA \\ Email:mahamed@uci.edu,dsenkal@uci.edu and andrei.shkel@uci.edu
}

\begin{abstract}
This paper reports the experimental demonstration of Quality factor (Q-factor) improvement of fused quartz hemispherical resonators after post-fabrication annealing. Several identical fused quartz 3D hemispherical resonators, thickness $1 \mathrm{~mm}$ and diameter $10 \mathrm{~mm}$ were fabricated using micro-glassblowing process and tested before and after annealing. Results showed that annealing at $800{ }^{\circ} \mathrm{C}$ for 6 hours followed by slow overnight cooling significantly improved $Q$ factor, from $666 \times 10^{3}$ to $932 \times 10^{3}$, in our experiments. The annealing dependence was investigated by varying temperature from $500{ }^{0} \mathrm{C}$ to $800{ }^{\circ} \mathrm{C}$. Change in Q-factor was observed to be minimal $(<10 \%)$, when annealed at $500{ }^{\circ} \mathrm{C}$, for both $\mathrm{n}=2$ and $\mathrm{n}=3$ wineglass modes. Q-factor changes were $\sim \mathbf{4 0} \%$, when annealed at $800{ }^{\circ} \mathrm{C}$. The experimental result on $\mathrm{Q}$-factor enhancement is valuable for the development of very high $Q$ fused quartz vibratory MEMS resonators for use in precision inertial sensing.
\end{abstract}

\section{INTRODUCTION}

A high Q-factor is desired in MEMS resonators, rate gyroscopes, RF filters, and clocks. High Q-factor is associated with low energy loss, allowing the resonator to oscillate longer without pumping additional energy in the system. Compared to other mechanical materials, fused quartz is a preferred resonator material due to its low internal thermo-elastic dissipation and its potential for achieving high Q-factor in resonating structures. For example, macro-scale fused quartz hemispherical resonators achieved over 25 million Q-factor [1], and research on fused silica gravitational wave detectors reported Q of 10 million [2]. These devices attracted many devoted efforts to produce fused silica resonators on microscale using MEMS-based technologies [3]-[6]. Wafer-scale micro-glassblown fused quartz resonators showed promise by demonstrating 3D MEMS hemispherical structures with low surface roughness and high symmetry [3].

Achieving high Q-factor in smaller devices, on the order of a millimeter, remains an intriguing prospect. Post-fabrication enhancements of Q-factor are important options in achieving higher $\mathrm{Q}$ and reducing energy losses. These possibilities have triggered some recent studies on prediction of Q-factor [7], studies to understand loss mechanism and possible improvement of Q-factor [8]. Among different energy loss mechanisms, losses due to chemical impurities, surface quality, intentional or unintentional surface coating, and hydration are some energy loss mechanisms [8], [12]-[13] that can be potentially improved in the post-fabrication manufacturing process.

Different techniques for enhancing Q-factor were used in the literature. For example, localized laser annealing on silicon micro-paddle oscillator improved Q to $1.4 \times 10^{6}$ at $470 \mathrm{kHz}$ [9]. Degenerate doping performed on silicon bulk acoustic micro resonator reduced its temperature coefficient of frequency and achieved Q of $28 \times 10^{3}$ at $\sim 105 \mathrm{MHz}$ [10]. Annealing of small scale fused quartz hemispherical resonators have not been sufficiently studied, although evidence of producing very high Q of $2 \times 10^{8}$ at $384 \mathrm{~Hz}$, after annealing at $950{ }^{\circ} \mathrm{C}$ on large scale (24-29cm in length) fused quartz rods were demonstrated in [11]. Surface damage in fused silica resonator due to polishing was demonstrated to improve after etching removal of $5 \mu \mathrm{m}$ of surface, and showed Q increase from $3 \times 10^{6}$ to $2 \times 10^{7}$ [8]. In addition, annealing of macro-scale hemispherical fused quartz structures predicted stress relaxation resulting in enhanced Qfactor [12].

This paper, for the first time, experimentally demonstrates the effect of post-fabrication annealing leading to Q-factor enhancement of fused quartz hemispherical micro-resonators.

\section{DESIGN AND FABRICATION}

Figure 1 shows the fabrication flow and sample of a fabricated device.

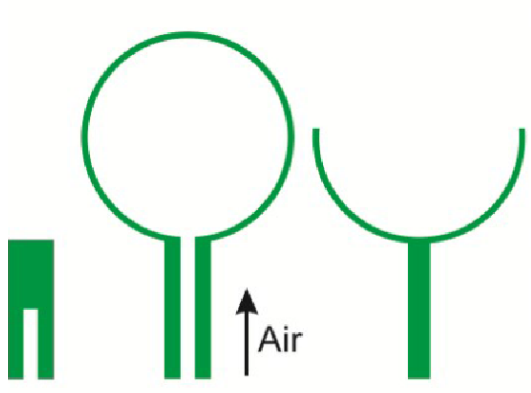

(a) (b)

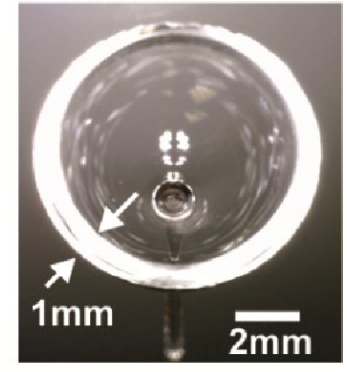

(d)
Figure 1. Fabrication flow of fused quartz wineglass resonator (a) softeing of quartz, (b) pressure difference, (c) trimming, and (d) fabricated device.

This work is supported by DARPA grant W31P4Q-11-1-0006 


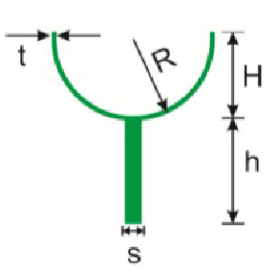

(a)

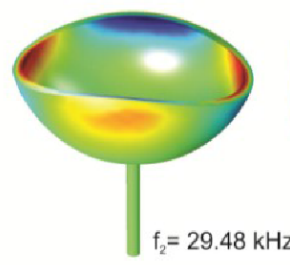

(b)

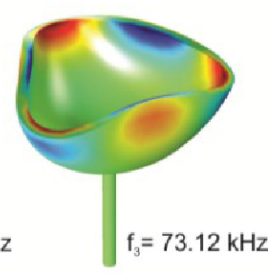

(c)
Figure 2. (a) Schematic of geometric parameters. (b)-(c) FEM results showing $\mathrm{n}=2$ and $\mathrm{n}=3$ modes of the resonator at $29.48 \mathrm{kHz}$ and $73.12 \mathrm{kHz}$.

We utilize glassblowing technique to fabricate 3D microhemispherical structures [3]. Our process relies on softening of quartz at its transitional temperature, and uses viscous deformation of quartz to define the 3D shell by pressure difference and surface tension. In this work, a glassblowing concept similar to [3] was used; however a blow-torch was utilized to raise the temperature of quartz to its softening point and then air is flown to create pressure difference that defines the spherical structure (Figure 1). Thin $(\sim 1 \mathrm{~mm})$ and $10 \mathrm{~mm}$ in diameter fused quartz wineglass devices were fabricated, as shown in Figure 1(d).

The resonance mode shapes, diameter, shell thickness, and stem diameter were numerically modeled, and results are summarized in Table I. Finite element analysis software COMSOL Multiphysics was used to model the 3-D wineglass shell structure with stem and to find its resonance frequencies and mode shapes. The mode shapes of interest were the highest displacement modes, $\mathrm{n}=2$ and $\mathrm{n}=3$, also known as wineglass modes, shown in Figure 2.

TABLE I. DEVICE DESIGN AND ESTIMATED RESONANCE FREQUENCIES

\begin{tabular}{c|ccccccc}
\hline \multirow{2}{*}{$\begin{array}{c}\text { Device } \\
\text { No }\end{array}$} & \multicolumn{4}{|c}{ Geometric Dimensions } & \multicolumn{3}{c}{$\begin{array}{c}\text { Estimated } \\
\text { Frequencies }\end{array}$} \\
\cline { 2 - 8 } & $\begin{array}{c}\boldsymbol{R} \\
(\mathbf{m m})\end{array}$ & $\begin{array}{c}\boldsymbol{H} \\
(\mathbf{m m})\end{array}$ & $\begin{array}{c}\boldsymbol{h} \\
(\mathbf{m m})\end{array}$ & $\begin{array}{c}\boldsymbol{s} \\
(\mathbf{m m})\end{array}$ & $\begin{array}{c}\boldsymbol{t} \\
(\mathbf{m m})\end{array}$ & $\begin{array}{c}\mathbf{N 2} \\
(\mathbf{k H z})\end{array}$ & $\begin{array}{c}\mathbf{N 3} \\
(\mathbf{k H z})\end{array}$ \\
\hline A & 11.5 & 4.4 & 13 & 1 & 0.8 & 34.31 & 82.68 \\
B & 11.5 & 5.5 & 18 & 1 & 1.1 & 30.79 & 76.73 \\
C & 11 & 4.8 & 16 & 0.9 & 1 & 31.67 & 77.03 \\
D & 12.5 & 6.5 & 23 & 0.8 & 1 & 29.48 & 73.12 \\
E & 12 & 4.6 & 14 & 0.9 & 1 & 32.11 & 78.61 \\
\hline
\end{tabular}

\section{EXPERIMENTAL RESULTS}

Experimental characterization was performed by mounting the resonator on a custom built piezo-actuator stage inside a vacuum chamber that is equipped with an optical port. Figure 3 shows the configuration and image of the setup. Devices were excited at its resonance by a piezo-actuator. A Laser Doppler Vibrometer (LDV) (Polytec OFV500), together with a network analyzer (Agilent 4395A) were used to measure the frequency responses. Details regarding the piezo-actuator, excitation mechanisms, and LDV sensing are available in our earlier work [14].

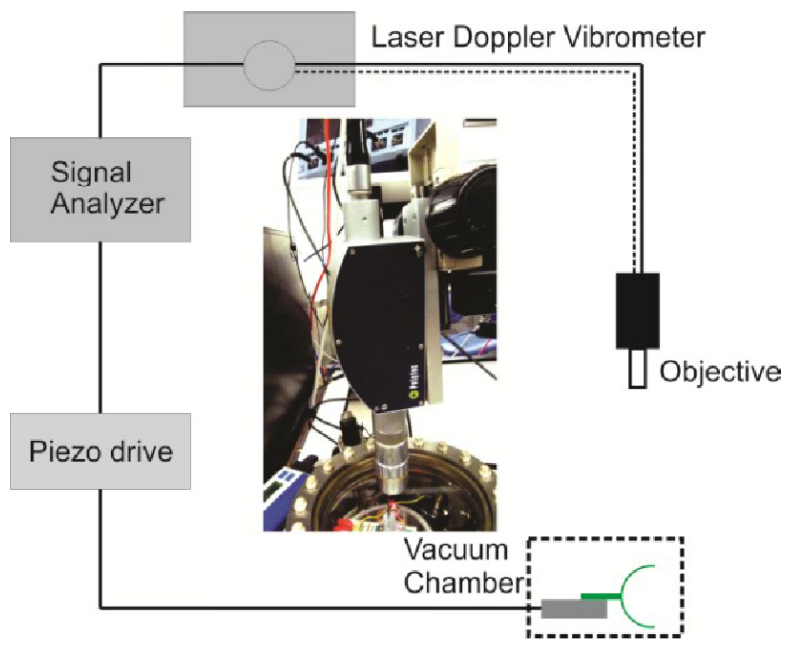

Figure 3. Schametic and picture showing the experimental configuration, vaccum chamber, and LDV.

Analysis was first carried out to identify measurement uncertainties from the experimental and mounting errors. Experiments were repeated multiple times for different mounting configurations, laser positioning, and for measurements repeatability. Response of the resonator was recorded in each run and compared for multiple runs to calculate the uncertainty in the experiment, while keeping the input excitation constant. Results showed that the cumulative experimental uncertainty was less than $5 \%$.

A total of five devices were tested, geometric parameters and estimated frequencies are listed in Table I. Annealing temperatures were varied from $500{ }^{\circ} \mathrm{C}$ to $800{ }^{\circ} \mathrm{C}$ for different annealing profiles between 4-6 hours, followed by slow overnight cooling to room temperature. Changes in Q-factor $(\Delta \mathrm{Q})$, before and after each annealing step for both $\mathrm{n}=2$ and $\mathrm{n}=3$ modes were calculated to identify percent of changes due to annealing. The $\Delta \mathrm{Q}$ was then normalized by $\mathrm{Q}$, in order to identify the changes on a same scale for all devices.

Figure 4 shows changes in normalized Q-factor $(\Delta \mathrm{Q} / \mathrm{Q})$ over annealing temperature, for both $n=2$ and $n=3$ modes. It shows improvements when annealed at temperatures over 500 ${ }^{0} \mathrm{C}$. The changes were larger when annealed at $700{ }^{\circ} \mathrm{C}$ and 800 ${ }^{0} \mathrm{C}$, as observed for both $\mathrm{n}=2$ and $\mathrm{n}=3$ modes, in our experiments. We believe, lowering of Q-factor can be attributed to the presence of water molecules that created hydration of the surface, together with other surface residues from fabrication and $\mathrm{OH}$ content in the silica matrix. Annealing improved these conditions. At higher temperature water dehydrates, fabrication residues ashes out, and $\mathrm{OH}$ content reduces. Mitrofanov and Tokmakov [13] studied temperature dependence on energy dissipation of fused silica fibers by heating sample up to $330{ }^{\circ} \mathrm{C}$. Their results suggested that water absorbed on the surface reduces Q-factor and it was improved after heating, mainly due to de-absorbing the surface by heating, [13] also suggested that water molecules can form silica-silanol ( $\mathrm{SiOH})$ strong bonds on the surface. 


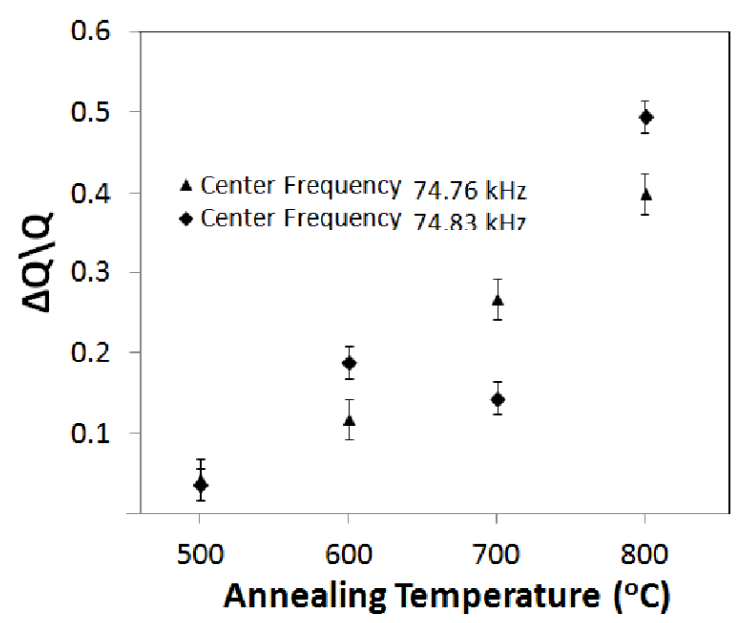

Figure 4. Changes in normalized $\mathrm{Q}$ are improved with increase in annealing temperatures.

We found that slow heating to annealing temperature (700-800 ${ }^{\circ} \mathrm{C}$ ) and staying at annealing temperature for 4-6 hours eliminates possible water molecules, fabrication residues, and reduces $\mathrm{OH}$ content from the silica matrix that in turns improves Q-factor.

Another important parameter for a resonator is the time constant $(\tau)$, which is related to the inverse of Q-factor $\left(\tau^{-1}=\right.$ $\pi^{*} f / Q, f$ is the resonance frequency) associated with energy dissipation via stem. The differences in time constants $\left(\tau_{l}\right.$ and $\tau_{2}$ ) along resonators two principal dissipation axes showed possible presence of defects, density variation, or presence of surface coatings, surface residues, machining defects, presence of residual gases in the chamber [8], [12]. Some of these causes can be corrected in the post-fabrication treatment. For example, the machining micro-defects created during polishing of fused quartz were minimized by chemical etching removal of thin layer of material from the surface, and resulted in improving $\mathrm{Q}$ from $3 \times 10^{6}$ to $2 \times 10^{7}$ [8].

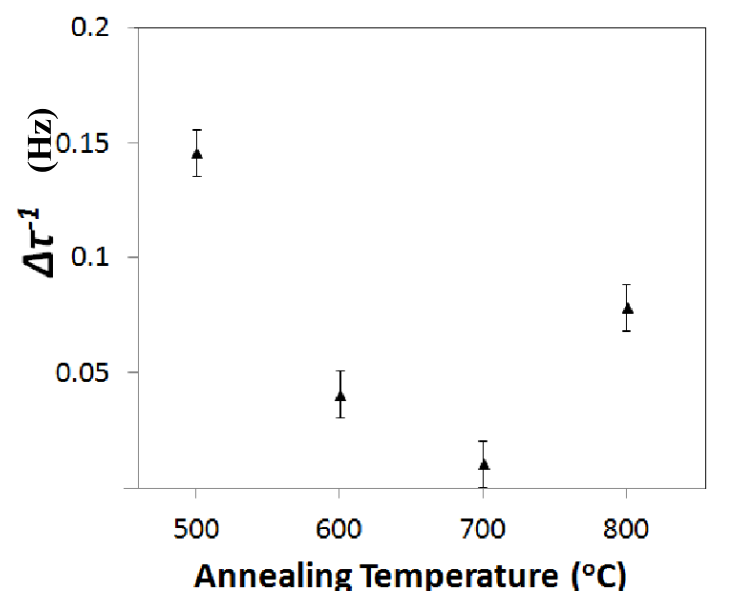

Figure 5. Changes of inverse of time constants $\left(\Delta \tau^{-1}\right)$ lowers with annealing temperature.

The inverse of $\Delta \tau^{-1}\left(\Delta \tau^{-1}=1 / \Delta \tau_{1}^{-1}-1 / \Delta \tau_{1}^{-1}\right)$ relates to the differences in energy dissipation along two principal dissipation axes. Our results showed that $\Delta \tau^{-1}$ lowers with most of the higher $\left(>500{ }^{0} \mathrm{C}\right)$ annealing temperatures, as shown in Figure 5. It reduces by orders of magnitude, for example from 0.146 to 0.011 , when annealing temperature rose from $500{ }^{\circ} \mathrm{C}$ to $700{ }^{\circ} \mathrm{C}$. We believe annealing helped in reducing the internal stress in the $3 \mathrm{D}$ shell structure. Likely, annealing also helped eliminating possible residues present on the surface from fabrication and handling, as well as helped recover edge roughness. In [15], we presented the results showing that annealing helps improving surface roughness. Annealing at higher temperature (e.g, $800{ }^{0} \mathrm{C}$ ) removed possible surface contamination from fabrication, which remained even after wet cleaning.

Table II and III summarize the results of four different devices, before and after annealing. $F_{1}$ and $F_{2}$ correspond to frequencies along two principal axes. Accordingly, $Q_{1}$ and $Q_{2}$ correspond to Q-factors along these two axes. The difference in $F_{1}$ and $F_{2}$ frequencies showed the fabrication asymmetry and the differences between $Q_{1}$ and $Q_{2}$ correspond to asymmetry in energy dissipation. After annealing, both $Q_{l}$ and $Q_{2}$ were improved. The frequencies were also shifted after annealing due to possible release of surface contaminants, water content, and impurities from silica matrix.

TABLE II. Q-FACTOR AND FREQUENCIES BEFORE ANNEALING

\begin{tabular}{ccccc}
\hline & \multicolumn{4}{c}{ Before Annealing } \\
\cline { 2 - 5 } Device & $\boldsymbol{f}_{\mathbf{1}}$ & $\boldsymbol{Q}_{1^{3}}$ & $\boldsymbol{f}_{2}$ & $\mathbf{Q}_{2}$ \\
& $(\boldsymbol{k H z})$ & $* 10^{3}$ & $(\boldsymbol{k H z})$ & $* 10^{3}$ \\
$\mathrm{~A}$ & 79.58 & 110.8 & 79.94 & 102.1 \\
B & 73.09 & 383.1 & 73.36 & 362.1 \\
$\mathrm{C}$ & 71.74 & 227.8 & 72.22 & 186.3 \\
D & 74.52 & 666.4 & 74.62 & 624.7 \\
\hline
\end{tabular}

TABLE III. Q-FACTOR AND FREQUENCIES AFTER ANNEALING

\begin{tabular}{|c|c|c|c|c|c|}
\hline \multirow[b]{2}{*}{ Device } & \multicolumn{4}{|c|}{ After Annealing } & \multirow{2}{*}{$\begin{array}{c}\text { Annealing } \\
\text { Temp } \\
\text { ('C) }\end{array}$} \\
\hline & $\begin{array}{c}f_{l} \\
(\boldsymbol{k} H z)\end{array}$ & $\underset{* 10^{3}}{Q 1}$ & $\begin{array}{c}f_{2} \\
(\boldsymbol{k H} ; z)\end{array}$ & $\underset{* 10^{3}}{Q_{2}}$ & \\
\hline A & 79.61 & 115.6 & 79.96 & 105.9 & 500 \\
\hline B & 73.13 & 427.9 & 73.39 & 430.2 & 600 \\
\hline C & 71.74 & 288.6 & 72.22 & 213.1 & 700 \\
\hline D & 74.58 & 932.2 & 74.68 & 933.5 & 800 \\
\hline
\end{tabular}

The optimum annealing profile improved Q-factor of a 10 $\mathrm{mm}$ diameter ( $1 \mathrm{~mm}$ thickness) resonator from $666 \times 10^{3}$ to almost a million $\left(932 \times 10^{3}\right)$, as shown in Figures 5 and Figure 6. The center frequencies are $74.523 \mathrm{kHz}$ and $74.57 \mathrm{kHz}$, before and after annealing. The Q-factor improvement remained unchanged over time. The results offer an 
opportunity for development of low cost, wafer-level manufacturing of high Q hemispherical resonator gyroscopes, clocks and inertial systems using fused quartz.

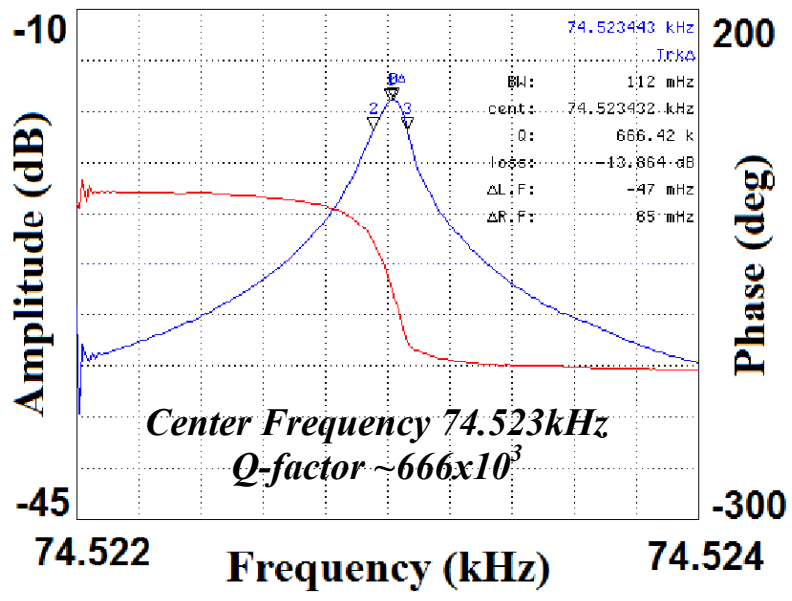

Figure 6. Frequency sweep before annealing, showing Q of $666.42 \mathrm{k}$ at $74.523 \mathrm{kHz}$ center frequency.

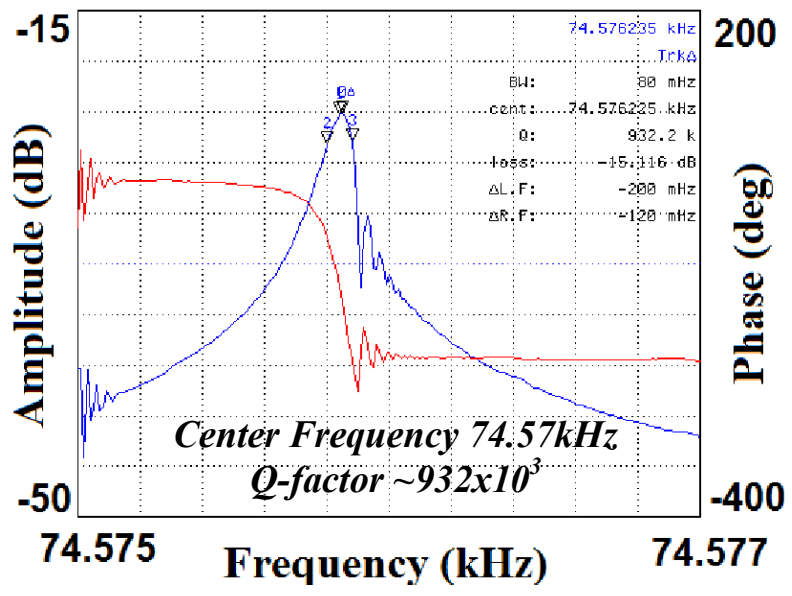

Figure 7. Frequency sweep after annealing, showing $\mathrm{Q}$ factor improvement by $40 \%$.

\section{CONCLUSIONS}

We have experimentally verified that annealing could significantly improve Q-factor and subsequently reduce energy loss in fused quartz hemispherical resonators. This method does not require removal or addition of materials; it is non-destructive and simple to implement. Our results showed that as fabricated Q-factor of $666 \times 10^{3}$ was improved to $932 \times 10^{3}$ after annealing at $800{ }^{0} \mathrm{C}$ for 6-hours with slow overnight cooling to room temperature. Improvement was noticed in multiple samples with consistency. Demonstration of Q-factor enhancement presented in this paper are envisioned to be utilized for development of high-Q MEMS resonators, which is necessary for implementation of high accuracy dynamic sensors, gyroscopes, RF filters, and clocks.

\section{ACKNOWLEDGMENT}

This material is based upon work supported by DARPA grant W31P4Q-11-1-0006 (Program Manager Dr. Robert Lutwak). The devices were designed, modeled and characterized in UCI MicroSystems Laboratory. Authors would like to acknowledge Jorg Meyer of UCI glass shop and UCI INRF staff Jake Hes, Mo Kebaili, Vu Phan and Lifeng Zheng.

\section{REFERENCES}

[1] D.M. Rozelle, "The hemispherical resonator gyro: from wineglass to the planets", in: Proc. AAS/AIAA Space Flight Mechanics Meeting, pp. 1157-1178, 2009

[2] W. J. Startin, M. A. Beilby, and P. R. Saulson, "Mechanical quality factors of fused silica resonators", Review of Scientific Instruments, vol. 69, no. 10, pp.3681, 1998.

[3] D. Senkal, M. J. Ahamed, A. A. Trusov, and A. M. Shkel, "High temperature micro-glassblowing process demonstrated on fused quartz and ULE TSG", Sensors and Actuators A: Physical, vol. 201, pp. 525531, October 2013.

[4] D. Senkal, M. J. Ahamed, A. A. Trusov and A. M. Shkel, "Achieving sub-Hz frequency symmetry in micro-glassblown wineglass resonators", in print, available online, IEEE Journal of Microelectromechanical Systems, 2013.

[5] M. J. Ahamed, D. Senkal, A. A. Trusov and A. M. Shkel, "Deep NLD plasma etching of glass", in Proceddings of IEEE Sensors 2013, pp. 1767-1770, November 4-6, Baltimore, Maryland, USA, 2013.

[6] J. Cho, J. Yan, J. A. Gregory, H. Eberhart, R. L. Peterson, and K. Najafi, "High-q fused silica birdbath and hemispherical 3-d resonators made by blow torch molding," Proceedings of IEEE MEMS 2013, pp. 177-180, Taipei, Taiwan, 2013.

[7] J. Choi, M. Cho, J. Rhim, "Efficient prediction of the quality factors of micromechanical resonators", Journal of Sound and Vibration, vol. 329, Issue 1, pp. 84-95, 2010.

[8] B. S. Lunin, S. N. Torbin, M. N. Danachevskaya, and I. V. Batov, "On the mechanism of acoustic losses in the hydroxylated surface layer of silica glass" Moscow University Chemistry Bulletin, vol. 42, no. 2, pp. 89, 2001.

[9] E. H. Haucke, X. Liu, J. F. Vignola, B. Houston, and J. W. Baldwin, "Effects of annealing and temperature on acoustic dissipation in a micromechanical silicon oscillator", Appl. Phys. Lett. 86, pp. 181903, 2005.

[10] A. K. Samarao and F. Ayazi, "Temperature compensation of silicon resonators via degenerate doping", IEEE Transactions on Electron Devices, vol.59, no.1, pp. 87-93, 2012.

[11] A. Ageev, B. C. Palmer, A. D. Felice, S. D Penn and P. R. Saulson, "Very high quality factor measured in annealed fused silica", Classical and Quantum Gravity, vol. 21 no. 16, pp. 3887, 2004.

[12] B. S. Lunin, "Physical and chemical bases for the development of hemispherical resonators for solid-state gyroscopes", Moscow, Russia: Moscow Aviation Institute, 2005.

[13] V.P. Mitrofanov and K.V. Tokmakov, "Effect of heating on dissipation of mechanical energy in fused silica fibers", Physics Letters A, vol. 308, pp. 212-218, 2003

[14] D. Senkal, M. J. Ahamed, A. A. Trusov, and A. M. Shkel, "Adaptable test-bed for characterization of micro-wineglass resonators," Proceedings of IEEE MEMS 2013, pp. 469-472, Taipei, Taiwan, Jan. 2013.

[15] M. J. Ahamed, D.k Senkal and A. M. Shkel, "Improvement of sidewall roughness in deep glass etched MEMS vibratory sensors", in print Proceedings of IEEE ISISS 2014, Laguna Beach, CA, USA, 25-26 February 2014. 